\section{Aus den Medizinischen Universitäten}

\section{Mitteilungen der Medizinischen Universität Wien}

Erteilung einer Lehrbefugnis als Privatdozent

An der Medizinischen Universität Wien wurde entsprechend den Bestimmungen des Universitätsorganisationsgesetzes 2002 die Lehrbefugnis erteilt an:

Dr. Ralf Herwig (Universitätsklinik für Urologie) für Urologie mit Datum vom 13. Jänner 2010.

Dr. Ilse Krebs (Krankenhaus Rudolfstiftung, Augenabteilung) für Augenheilkunde und Optometrie mit Datum vom 13. Jänner 2010.

Dr. Christian Scheuba (Universitätsklinik für Chirurgie) für Chirurgie mit Datum vom 13. Jänner 2010.

Dr. Dietmar Tamandl (Universitätsklinik für Chirurgie) für Chirurgie mit Datum vom 13. Jänner 2010.

Dr. Jasmin Arrich (Universitätsklinik für Notfallmedizin) für Notfallmedizin mit Datum vom 20. Jänner 2010.

Mag. Dr. Johann Lehrner (Universitätsklinik für Neurologie) für Medizinische Psychologie mit Datum vom 20. Jänner 2010.

Dr. Rudolf Paul Obermayr (SMZ-Ost, 3. Medizinische Abteilung) für Innere Medizin mit Datum vom 20. Jänner 2010.
Dr. Elisabeth Stögmann (Universitätsklinik für Neurologie) für Neurologie mit Datum vom 20. Jänner 2010.

Mag. Dr. Merima Bublin (Zentrum für Pathophysiologie, Infektiologie und Immunologie, Institut für Pathophysiologie) für Molekulare Allergologie mit Datum vom 25. Jänner 2010.

Dr. Dominik Georg Haider (Universitätsklinik für Innere Medizin III) für Klinische Pharmakologie mit Datum vom 25. Jänner 2010.

Dr. Rupert Lanzenberger (Universitätsklinik für Psychiatrie und Psychotherapie) für Neurosciences mit Datum vom 25. Jänner 2010.

Dr. Luc Snyers (Zentrum für Anatomie) für Molekularbiologie mit Datum vom 25. Jänner 2010.

Dr. Christoph Arnoldner (Universitätsklinik für Hals-, Nasen- und Ohrenkrankheiten) für Hals-, Nasen- und Ohrenheilkunde mit Datum vom 27. Jänner 2010.

Dr. Valentin Fuhrmann (Universitätsklinik für Innere Medizin III) für Innere Medizin mit Datum vom 27. Jänner 2010.

Dr. Georg-Christian Funk (Otto Wagner Spital, 1. Interne Lungenabteilung) für Innere Medizin mit Datum vom 27. Jänner 2010.

Dr. Philipp Peloschek (Universitätsklinik für Radiodiagnostik) für Medizinische Radiologie-Diagnostik mit Datum vom 27. Jänner 2010.

Dr. Michael Saletu (Neurologisches Rehabilitationszentrum Rosenhügel) für Neurologie mit Datum vom 27. Jänner 2010. 